\title{
Analysis and Correlation among Agronomic and Weather Parameters in Barley (Hordeum vulgare L.) Cultivars under Semi-Arid Regions of Haryana, India
}

\author{
Karan Chhabra*, Mahendra Bele, Raj Singh and Anil Kumar \\ Department of Agricultural Meteorology, \\ College of Agriculture (COA), CCS Haryana Agricultural University, \\ Hisar-125004, Haryana, India \\ *Corresponding author
}

Keywords

Rabi barley, Agronomic and weather components, Growing environment, Correlation analysis

Article Info

Accepted:

12 April 2019

Available Online:

10 May 2019

\section{A B S T R A C T}

A study was carried out in 2016-17 and 2017-18 growing seasons with 4 different "barley" cultivars viz., $\mathrm{V}_{1}-\mathrm{BH} 393, \mathrm{~V}_{2}-\mathrm{BH} 902, \mathrm{~V}_{3}-\mathrm{BH} 946$ and $\mathrm{V}_{4}-\mathrm{BH} 885$ sown under different growing environments, i.e., $\mathrm{D}_{1}-3^{\text {rd }}$ November; $\mathrm{D}_{2}-18^{\text {th }}$ November; $\mathrm{D}_{3}-3^{\text {rd }}$ December and $\mathrm{D}_{4^{-}} 19^{\mathrm{th}}$ December. The correlations among growth, weather and agrometeorological parameters, yield and yield attributes, soil moisture and canopy temperature were investigated using correlation analysis during vegetative and reproductive phases of crop. Yield studies had positive association with growth parameters during both phases. All growth parameters had shown significant correlation during reproductive phase as compare to vegetative phase. The agrometeorological indices like GDD, HTU, PTU, RTD, and RUE were highly correlated during reproductive phase and HUE at vegetative phase with weather parameters. Maximum temperature, BSS and RH (M) had positive correlation while minimum temperature, RH (E), WS, PE and RF had a negative relationship with yield and its attributes except straw yield during reproductive phase. The association of growth parameters with agrometeorological indices and yield attributes with agrometeorological indices was higher at vegetative phase than reproductive phase. Canopy temperature was found significant relationship with soil moisture of depth 0-15 and 15-30 cm during both the barley crop seasons under different growing environments. It was concluded that these characteristics could be important selection criteria to quantify crop-weather relationships in barley crop under different growing environments.

\section{Introduction}

Barley is the major source of food for large population of cool and semi-arid areas of the world, where wheat and other cereals are less adapted. Barley grain is a staple in Tibetan cuisine and was eaten widely by peasants in
Medieval Europe. Grown on an area of 35 thousand hectares with a productivity of 3418 $\mathrm{kg} / \mathrm{ha}$ (Agriharyana, 2017-18) is an important rabi crop in Haryana. Barley is a short growing season crop and has good drought and salinity tolerance. It is productive under adverse environments than other cereals. In 
India, it is cultivated as a winter crop in tropical regions and as a summer crop in temperate region. Rainfall, temperature and radiation are meteorological parameters, which influence all aspects and stages of crop growth in barley. Solar radiation interception and its efficiency play major role in dry matter production of barley. Slight increase in minimum temperature can encourage respiration rate and water requirement and slow down the assimilation rate during growth and development in barley. Correlation analysis is a method of statistical evaluation used to study in agriculture to strengthen the relationship between crop and weather parameters which are numerically measured with application of continuous variables (e.g. plant height, dry weight, temperature and rainfall). This particular type of analysis is useful for a researcher to establish possible connections between those variables (Panse and Sukhatme, 1985) to analysis and determines cause and effect of particular cultivar in a defined environment. Samarrai et al., (1987) suggested that barley genotypes, which are long-day plants, are varied in estimates of phenological growth and agronomic features in accordance with different growing environments. Dissimilarities in reproductive period among barley cultivars were revealed primarily because of the differences in vegetative period comparatively than in days to physiological maturity. Days to maturity is positively correlated with extent of the vegetative phase in barley. Harvest index differed markedly among barley cultivars with a mean value of $27 \%$ and a range of 19 to $42 \%$. A positive correlation was observed between plant height and grain yield in barley by Hadjichristodoulou (1987). Whereas Shephred et al., (1987) noticed an intense linear correlation between grain yield or total dry matter yield and number of grains per plant. Anthesis phase was concluded to be negatively correlated with yield among
(Shephred et al., 1987) barley (Hadjichristodoulou, 1987) genotypes. Days from planting to maturity, plant height, root, and shoot dry matter yield; each have a negative correlation with harvest index in cereal crops (Ehdaie and Waines, 1993). Shamim (2013) indicated that in cereal crops grain filling duration had a positive and significant correlation with the grain yield and flowering duration (FD) had positive and significant correlation with grain yield and total biomass accumulation of the cultivars. However, soil moisture exhibited significant effects on yield components of barley cultivars. Moreover, a negative yield response was produced by decreasing irrigation frequency and increasing salinity in water available to soil (Campbell, 1980). Correlation analyses among grain yield with all the yield components in Two-Rowed of Barley (Hordeum vulgare convar. distichon) Varieties indicated that grain yield was positively and significantly associated with all the yield components except 1000-kernel weight. The highest correlation coefficients were found between kernel number per spike and grain yield, and between harvest index and grain yield (Carpici and Celik, 2012).

\section{Materials and Methods}

For determining the correlations between agronomic and weather parameters in barley the field experiment was conducted during Rabi season of 2016-17 and 2017-18 at Research Farm of Department of Agricultural Meteorology, CCSHAU; Hisar located at latitude $29^{\circ} 10^{\prime} \mathrm{N}$, longitude $75^{\circ} 46^{\prime} \mathrm{E}$ and altitude of $215.2 \mathrm{~m}$ above mean sea level, to quantify crop-weather relationships under different growing environments. The weekly values of weather parameters prevailed during both the seasons were worked out on the basis of daily data recorded at Agromet Observatory, CCS HAU, Hisar situated just beside the experimental site along with 
normal values for various weather parameters. The mean maximum temperature at the time of sowing was $30.1{ }^{\circ} \mathrm{C}$ and declined to lowest value $22.7^{\circ} \mathrm{C}$ during jointing and then again rose to $39.9^{\circ} \mathrm{C}$ consistently at physiological maturity during crop season 2016-17 while during $2017-18$, values ranged from $16.9^{\circ} \mathrm{C}$ to $42.9^{\circ} \mathrm{C}$. Comparisons between two crops seasons showed that mean maximum temperature prevailed from emergence physiological maturity was higher during 2016-17 as compared to subsequent crop season. Weekly morning relative humidity value during cropseason 2016-17 was 94\% during sowing then it fluctuated throughout crop season but lowest value was observed during reproductive phase with a lowest value of $57 \%$ at physiological maturity. However, during 2017-18, it was $99 \%$ coincided with emergence and at jointing and lowest of $45.3 \%$ at physiological maturity. The crop season of 2016-17 received a total of $30.5 \mathrm{~mm}$ rainfall with 4 rainy days out of which vegetative phase received no rainfall however; only four rainy days were recorded during whole crop season 2017-18. The vegetative and the reproductive phase received a total of $45.0 \mathrm{~mm}$ and $7.5 \mathrm{~mm}$ rainfall, respectively, in 2017-18. The mean weekly wind speed values remained in the range of 1.2 to $5.6 \mathrm{~km} \mathrm{~h}^{-1}$ during first crop season However, this range was 1.0 to $5.0 \mathrm{~km}$ $\mathrm{h}^{-1}$ during subsequent crop season. The soils of Hisar are derived from Indo-Gangetic alluvium, which are very deep and sandy loam in texture its $\mathrm{pH}$ is alkali (8.1) with low organic matter $(0.43 \%)$ and high quantities of usable $\mathrm{N}$ and $\mathrm{K}$ in $\mathrm{kg} \mathrm{ha}^{-1}$.

The main plot treatments consisted of four dates of sowing $\left(D_{1}-3^{\text {rd }}\right.$ November, $D_{2}-18^{\text {th }}$ November, $\mathrm{D}_{3} 3^{\text {rd }}$ December and $\mathrm{D}_{4^{-}} 19^{\text {th }}$ December) and subplot treatments consisted four varieties $\left(\mathrm{V}_{1^{-}} \mathrm{BH} 393, \mathrm{~V}_{2^{-}} \mathrm{BH} 902, \mathrm{~V}_{3^{-}}\right.$ $\mathrm{BH}$ 946, $\left.\mathrm{V}_{4}-885\right)$ using split plot design. The unit plot size was $5.0 \times 3.6 \mathrm{~m}^{2}$ having Inter- row spacing of $22.5 \mathrm{~cm}$, respectively. The soil was medium in available $\mathrm{N}$ (162 kg/ha), lower in available $\mathrm{P}(25 \mathrm{~kg} / \mathrm{ha})$, and higher in available K (321 kg/ha). Sowing was done in rows spaced $22.5 \mathrm{~cm}$ apart at a depth of $5 \mathrm{~cm}$ with hand-pulled plough employing pora method. A fertilizer dose of 150:65:25 $\mathrm{kg} \mathrm{ha}^{-1}$ $\mathrm{N}, \mathrm{P}_{2} \mathrm{O}_{5}$ and $\mathrm{Zn}$ applied in the form of urea, triple super phosphate and zinc sulphate, respectively. Irrigation was applied at CRI stage to all treatments whereas, at tillering irrigation was applied to $\mathrm{D}_{4}\left(19^{\text {th }}\right.$ December $)$ only during the crop season. Each irrigation was of $6 \mathrm{~cm}$ depth. The coefficient of correlation was computed by dividing the sum of the product of deviations from the mean by the square root of the product of sum of squares of deviations from the respective mean of the two variables (Panse and Sukhatme, 1985) and its significance was tested at $5 \%$ levels of significance.

\section{Results and Discussion}

\section{Correlation studies between growth parameters and yield and yield attributes}

All the growth parameters were negatively associated with spike length, no. of spikelets per spike and no. of grains per spike during vegetative and reproductive phase except in plant height which was positively associated with no. of spikelets per spike and no. of grains per spike at vegetative and reproductive phase (Table 1). All other yield and yield attributes like no. of effective tillers, test weight, grain yield, biological yield, straw yield and harvest index had positive association with growth parameters during vegetative and reproductive phase. The association of growth parameters like plant height, dry matter and no. of tillers with yield and yield attributes was higher during reproductive phase than vegetative phase, whereas, among growth parameters the association of LAI with yield and yield 
attributes was higher during vegetative phase than reproductive phase except in association with no. of spikelets per spike and no, of grains per spike where, it was higher during reproductive phase than vegetative phase.

\section{Correlation studies between weather and growth parameters}

The results of correlation studies between growth and weather parameters at vegetative and reproductive phase of development of crop are given in Table 2. All growth parameters viz. plant height, no. of tillers, dry matter (DM) and leaf area index (LAI) were negatively associated with maximum and minimum temperature, bright sunshine hours and pan evaporation during vegetative phase whereas, growth parameters were positively correlated with morning and evening relative humidity, wind speed and cumulative rainfall during vegetative phase. During reproductive phase, all growth parameters were either positively or negatively associated with weather parameters. All growth parameters were shown significantly correlation during reproductive phase as compare to vegetative phase.

\section{Correlation studies of the weather parameters with agrometeorological indices}

All agrometeorological indices were negatively associated with morning and evening relative humidity during reproductive phase and with pan evaporation at vegetative phase whereas; all agrometeorological indices were positively correlated with morning and evening relative humidity and cumulative rainfall at vegetative phase and with maximum temperature and bright sunshine hours at reproductive phase (Table 3). At vegetative and reproductive phase, all agrometeorological indices were either positively or negatively associated with other weather parameters. The agrometeorological indices like GDD, HTU, PTU, RTD, and RUE were highly correlated during reproductive phase than vegetative phase. Relationship of HUE with weather parameters was higher at vegetative phase than reproductive phase.

\section{Correlation studies between weather parameters with yield and yield attributes}

During vegetative phase, all the yield parameters were found positively associated with maximum temperature, minimum temperature, BSS and PE except with straw yield. Biological yield attributes and yield parameters were negatively correlated with wind speed, morning and evening relative humidity except straw yield. Rainfall had negative association with barley yield components. Both straw and biological yield had significant relationship with all the weather parameters with positive and negative values at vegetative phase (Table 4).

During reproductive phase, barley crop had positive correlation with maximum temperature, while negative relationship with minimum temperature except straw yield. BSS and RH (M) showed positive relationship with all the yield components of barley crop except straw yield. RH (E), WS, $\mathrm{PE}$ and RF had negative correlation with yield and its attributes of barley crop. while straw yield shown positive correlation with WS, PE and RF. Grain yield had significantly positive relationship with maximum temperature, $\mathrm{RH}$ (M), BSS, WS, PE and RF.

\section{Correlation studies between growth parameters with agrometeorological indices}

All the growth parameters had positive significant association with agrometeorological indices during vegetative 
phase (Table 5). The growth parameters like dry matter and plant height had positive significant association with agrometeorological indices during reproductive phase. LAI had negative relationship with all agromet indices. No. of tillers also found negative correlation with all agromet indices except with RUE and HUE. The association of growth parameters like plant height, dry matter, LAI and no. of tillers with agrometeorological indices was higher at vegetative phase than reproductive phase.

Table.1 Correlation coefficient of growth parameters with yield and yield attributes during vegetative and reproductive phase under different growing environments

\begin{tabular}{|c|c|c|c|c|c|c|c|c|c|}
\hline Particulars & $\begin{array}{c}\text { No. of } \\
\text { effective } \\
\text { tillers }\end{array}$ & $\begin{array}{c}\text { Spike } \\
\text { length } \\
\text { (cm) }\end{array}$ & $\begin{array}{c}\text { No. of } \\
\text { spikelets/ } \\
\text { spike }\end{array}$ & $\begin{array}{c}\text { No. of } \\
\text { grains/ } \\
\text { spike }\end{array}$ & $\begin{array}{c}\text { Test } \\
\text { weight } \\
\text { (g) }\end{array}$ & $\begin{array}{c}\text { Grain } \\
\text { yield } \\
\text { (q/ha) }\end{array}$ & $\begin{array}{c}\text { Biological } \\
\text { yield } \\
\text { (q/ha) }\end{array}$ & $\begin{array}{c}\text { Straw } \\
\text { yield } \\
\text { (q/ha) }\end{array}$ & $\begin{array}{c}\text { HI } \\
\mathbf{\%}\end{array}$ \\
\hline LAI & 0.15 & -0.02 & -0.03 & -0.01 & 0.10 & 0.11 & 0.13 & 0.07 & 0.07 \\
\hline DM & 0.17 & -0.04 & -0.04 & -0.01 & 0.12 & 0.11 & 0.14 & 0.09 & 0.05 \\
\hline Plant height & 0.15 & -0.09 & 0.16 & $0.20^{*}$ & 0.08 & $0.19^{*}$ & 0.15 & 0.03 & 0.17 \\
\hline No. of tillers & 0.14 & -0.03 & -0.03 & -0.01 & 0.10 & 0.11 & 0.12 & 0.06 & 0.07 \\
\hline \multicolumn{7}{|c|}{ Reproductive phase } \\
\hline LAI & 0.13 & -0.01 & -0.05 & -0.03 & 0.09 & 0.07 & 0.10 & 0.07 & 0.03 \\
\hline DM & $0.28^{*}$ & -0.05 & -0.08 & -0.04 & $0.19^{*}$ & $0.18^{*}$ & $0.23^{*}$ & 0.14 & 0.10 \\
\hline Plant height & $0.33^{*}$ & $-0.34^{*}$ & $0.38^{*}$ & $0.45^{*}$ & $0.18^{*}$ & $0.34^{*}$ & $0.29^{*}$ & 0.02 & $0.29^{*}$ \\
\hline No. of tillers & $0.44^{*}$ & -0.02 & -0.06 & -0.02 & $0.29^{*}$ & $0.36^{*}$ & $0.39^{*}$ & $0.16^{*}$ & $0.25^{*}$ \\
\hline
\end{tabular}

$*$ Significance at $\mathrm{P}=0.05, \mathrm{LAI}=$ Leaf area index, $\mathrm{DM}=$ Dry matter, $\mathrm{HI}=$ Harvest index

Table.2 Correlation coefficient of weather parameters with growth parameters during vegetative and reproductive phase under different growing environments

\begin{tabular}{|c|c|c|c|c|}
\hline Particulars & Plant height & No. of tillers & DM & LAI \\
\hline \multicolumn{5}{|c|}{ Vegetative phase } \\
\hline Max. Temp. & -0.15 & $-0.22 *$ & $-0.28 *$ & $-0.25 *$ \\
\hline Min. Temp. & -0.15 & $-0.24 *$ & $-0.28 *$ & $-0.25 *$ \\
\hline RH (M) & 0.11 & 0.17 & $0.22 *$ & $0.19 *$ \\
\hline RH (E) & 0.14 & $0.21 *$ & 0.26 & $0.23 *$ \\
\hline WS & $0.21 *$ & $0.27 *$ & $0.32 *$ & $0.30 *$ \\
\hline BSS & -0.09 & -0.14 & $-0.20 *$ & $-0.17 *$ \\
\hline $\mathbf{P E}$ & -0.10 & $-0.18 *$ & $-0.23 *$ & $-0.20 *$ \\
\hline $\mathbf{R F}$ & $0.33 *$ & $0.32 *$ & $0.41 *$ & $0.39 *$ \\
\hline \multicolumn{5}{|c|}{ Reproductive phase } \\
\hline Max. Temp. & $0.34^{*}$ & $-0.41 *$ & $0.45^{*}$ & $-0.48 *$ \\
\hline Min. Temp. & $0.35^{*}$ & $-0.71 *$ & $0.57 *$ & $-0.68 *$ \\
\hline RH (M) & $-0.33 *$ & $0.55^{*}$ & $-0.48 *$ & $0.57 *$ \\
\hline RH (E) & $-0.40 *$ & $0.39 *$ & $-0.49 *$ & $0.50^{*}$ \\
\hline WS & 0.04 & $-0.41 *$ & $0.19 *$ & $-0.28 *$ \\
\hline BSS & $0.33^{*}$ & $-0.37 *$ & $0.42 *$ & -0.45 \\
\hline $\mathbf{P E}$ & $0.37 *$ & $-0.75^{*}$ & 0.60 & $-0.73 *$ \\
\hline RF & 0.13 & $-0.41 *$ & $0.26^{*}$ & $-0.31 *$ \\
\hline
\end{tabular}

$*$ Significance at $\mathrm{P}=0.05, \mathrm{LAI}=$ Leaf area index, $\mathrm{DM}=$ Dry matter,

Max. Temp. = Maximum temperature, Min. Temp. = Minimum temperature, $\mathrm{RH}(\mathrm{M})=$ Morning relative humidity, $\mathrm{RH}(\mathrm{E})=$ Evening relative humidity, WS $=$ Wind speed, $\mathrm{BSS}=$ Bright sun shine hour, $\mathrm{PE}=\mathrm{Pan}$ evaporation and $\mathrm{RF}=\mathrm{Rainfall}$ 
Table.3 Correlation coefficient of weather parameters with agrometeorological indices during vegetative and reproductive phase under different growing environments

\begin{tabular}{|c|c|c|c|c|c|c|}
\hline Particulars & GDD & HTU & PTU & RTD & RUE & HUE \\
\hline \multicolumn{7}{|c|}{ Vegetative phase } \\
\hline Max. Temp. & 0.03 & -0.02 & 0.03 & $-0.41 *$ & -0.13 & $-0.29 *$ \\
\hline Min. Temp. & 0.02 & -0.08 & 0.03 & $-0.41 *$ & -0.11 & $-0.27 *$ \\
\hline RH (M) & $0.03 *$ & $0.21 *$ & 0.02 & $0.28 *$ & 0.03 & $0.18^{*}$ \\
\hline RH (E) & $0.02 *$ & 0.16 & 0.02 & $0.35 *$ & 0.07 & $0.25^{*}$ \\
\hline WS & $0.02 *$ & -0.04 & 0.02 & $0.43 *$ & $0.18^{*}$ & $0.31 *$ \\
\hline BSS & 0.05 & 0.03 & 0.04 & $-0.31 *$ & -0.08 & $-0.25 *$ \\
\hline $\mathbf{P E}$ & -0.01 & $-0.19 *$ & -0.01 & $-0.31^{*}$ & -0.05 & $-0.23 *$ \\
\hline $\mathbf{R F}$ & 0.15 & 0.07 & 0.15 & $0.45^{*}$ & $0.29 *$ & $0.32 *$ \\
\hline \multicolumn{7}{|c|}{ Reproductive phase } \\
\hline Max. Temp. & $0.59 *$ & $0.64 *$ & $0.57 *$ & $0.31 *$ & 0.12 & 0.06 \\
\hline Min. Temp. & $0.84 *$ & $0.86^{*}$ & $0.87 *$ & $0.55^{*}$ & -0.12 & -0.11 \\
\hline RH (M) & -0.62 & $-0.59 *$ & $-0.64 *$ & $-0.54 *$ & -0.05 & -0.04 \\
\hline RH (E) & -0.54 & $-0.55^{*}$ & $-0.51 *$ & $-0.40 *$ & $-0.24 *$ & $-0.19 *$ \\
\hline WS & 0.31 & $0.31 *$ & $0.37 *$ & $0.35^{*}$ & $-0.34 *$ & -0.12 \\
\hline BSS & $0.50 *$ & $0.55^{*}$ & $0.48 *$ & $0.33 *$ & 0.10 & 0.05 \\
\hline PE & $0.81 *$ & $0.87 *$ & $0.84 *$ & $0.60 *$ & -0.13 & -0.01 \\
\hline RF & $0.41 *$ & $0.37 *$ & $0.46^{*}$ & $0.40 *$ & $-0.24 *$ & -0.15 \\
\hline
\end{tabular}

*Significance at $\mathrm{P}=0.05$

Max. Temp. $=$ Maximum temperature, Min. Temp. $=$ Minimum temperature, $\mathrm{RH}(\mathrm{M})=$ Morning relative humidity, $\mathrm{RH}(\mathrm{E})=$ Evening relative humidity, $\mathrm{WS}=$ Wind speed, $\mathrm{BSS}=$ Bright sun shine hour, $\mathrm{PE}=\mathrm{Pan}$ evaporation and $\mathrm{RF}=\mathrm{Rainfall}$

Table.4 Correlation coefficient of weather parameters with yield and yield attributes during vegetative and reproductive phase under different growing environments

\begin{tabular}{|c|c|c|c|c|c|c|c|c|c|}
\hline Particulars & $\begin{array}{c}\text { No. of } \\
\text { effective } \\
\text { tillers }\end{array}$ & $\begin{array}{c}\text { Spike } \\
\text { length } \\
(\mathrm{cm})\end{array}$ & $\begin{array}{c}\text { No. of } \\
\text { spikelets/ } \\
\text { spike }\end{array}$ & $\begin{array}{c}\text { No. of } \\
\text { grains/ } \\
\text { spike }\end{array}$ & $\begin{array}{c}\text { Test } \\
\text { weight } \\
\text { (g) }\end{array}$ & $\begin{array}{c}\text { Grain } \\
\text { yield } \\
\text { (q/ha) }\end{array}$ & $\begin{array}{c}\text { Biological } \\
\text { yield } \\
\text { (q/ha) }\end{array}$ & $\begin{array}{c}\text { Straw } \\
\text { yield } \\
\text { (q/ha) }\end{array}$ & HI \% \\
\hline \multicolumn{10}{|c|}{ Vegetative phase } \\
\hline Max. Temp. & $0.48 *$ & $0.26 *$ & $0.20 *$ & $0.30 *$ & $0.25^{*}$ & $0.78 *$ & $0.55 *$ & -0.13 & $0.75^{*}$ \\
\hline Min. Temp. & $0.45 *$ & $0.26 *$ & $0.18 *$ & $0.29 *$ & $0.25 *$ & $0.74 *$ & $0.56^{*}$ & -0.06 & $0.67 *$ \\
\hline RH (M) & $-0.35^{*}$ & $-0.18 *$ & -0.10 & -0.17 & $-0.21 *$ & $-0.49 *$ & $-0.33 *$ & 0.11 & $-0.49 *$ \\
\hline RH (E) & $-0.43 *$ & $-0.21 *$ & -0.15 & $-0.23 *$ & $-0.24 *$ & $-0.64 *$ & $-0.42 *$ & 0.16 & $-0.65^{*}$ \\
\hline WS & $-0.47 *$ & $-0.25^{*}$ & $-0.18^{*}$ & $-0.29 *$ & $-0.23 *$ & $-0.77 *$ & $-0.54 *$ & 0.14 & $-0.74 *$ \\
\hline BSS & $0.39 *$ & $0.19 *$ & 0.15 & $0.22 *$ & $0.20 *$ & $0.63^{*}$ & $0.38 *$ & $-0.20 *$ & $0.66 *$ \\
\hline PE & $0.38 *$ & $0.19 *$ & 0.13 & $0.20 *$ & 0.22 & $0.56^{*}$ & $0.38 *$ & -0.12 & $0.56 *$ \\
\hline RF & $-0.28 *$ & -0.17 & -0.09 & $-0.18 *$ & -0.15 & $-0.45^{*}$ & $-0.37 *$ & -0.01 & $-0.38 *$ \\
\hline \multicolumn{10}{|c|}{ Reproductive phase } \\
\hline Max. Temp. & 0.05 & 0.08 & 0.08 & 0.11 & 0.02 & $0.18^{*}$ & $0.20 *$ & 0.09 & 0.10 \\
\hline Min. Temp. & $-0.21 *$ & -0.08 & -0.07 & -0.10 & -0.10 & $-0.32 *$ & -0.15 & $0.18 *$ & $-0.38 *$ \\
\hline RH (M) & 0.09 & 0.05 & 0.03 & 0.07 & 0.00 & $0.20 *$ & 0.11 & -0.08 & $0.20 *$ \\
\hline RH (E) & -0.11 & -0.09 & -0.10 & -0.12 & $-0.07 *$ & $-0.23^{*}$ & $-0.21 *$ & -0.04 & $-0.19 *$ \\
\hline WS & $-0.38 *$ & $-0.24 *$ & $-0.19 *$ & $-0.29 *$ & $-0.19 *$ & $-0.70^{*}$ & $-0.53 *$ & 0.05 & $-0.64 *$ \\
\hline BSS & 0.04 & $0.06^{*}$ & 0.07 & $0.08^{*}$ & $0.01 *$ & $0.18^{*}$ & 0.13 & -0.01 & $0.16^{*}$ \\
\hline PE & $-0.24 *$ & $-0.12 *$ & -0.06 & -0.12 & -0.11 & $-0.37 *$ & $-0.24 *$ & 0.10 & $-0.37 *$ \\
\hline RF & $-0.27 *$ & -0.17 & -0.16 & $-0.21 *$ & -0.14 & $-0.51 *$ & $-0.38 *$ & 0.06 & $-0.49 *$ \\
\hline
\end{tabular}

*Significance at $\mathrm{P}=0.05$

Max. Temp. $=$ Maximum temperature, Min. Temp. $=$ Minimum temperature, $\mathrm{RH}(\mathrm{M})=$ Morning relative humidity, $\mathrm{RH}(\mathrm{E})=$ Evening relative humidity, $\mathrm{WS}=$ Wind speed, $\mathrm{BSS}=$ Bright sun shine hour, $\mathrm{PE}=\mathrm{Pan}$ evaporation and $\mathrm{RF}=\mathrm{Rainfall}$ 
Table.5 Correlation coefficient of growth parameters with agrometeorological indices during vegetative and reproductive phase under different growing environments

\begin{tabular}{|l|c|c|c|c|}
\hline \multicolumn{2}{|l|}{ Particulars } & LAI & DM & \multicolumn{2}{c|}{ Plant height } & No. of tillers \\
\hline \multicolumn{4}{|c|}{ Vegetative phase } \\
\hline HTU & $0.91^{*}$ & $0.89^{*}$ & $0.92^{*}$ & $0.88^{*}$ \\
\hline PTU & $0.83^{*}$ & $0.80^{*}$ & $0.82^{*}$ & $0.81^{*}$ \\
\hline RTD & $0.91^{*}$ & $0.89^{*}$ & $0.92^{*}$ & $0.88^{*}$ \\
\hline RUE & $0.96^{*}$ & $0.94^{*}$ & $0.92^{*}$ & $0.92^{*}$ \\
\hline HUE & $0.90^{*}$ & $0.93^{*}$ & $0.87^{*}$ & $0.85^{*}$ \\
\hline \multicolumn{7}{|l|}{} & $0.92^{*}$ & $0.83^{*}$ & $0.80^{*}$ \\
\hline GDD & $0.86^{*}$ & Reproductive phase & & $-0.84^{*}$ \\
\hline HTU & $-0.91^{*}$ & $0.86^{*}$ & $0.65^{*}$ & $-0.84^{*}$ \\
\hline PTU & $-0.87^{*}$ & $0.79^{*}$ & $0.55^{*}$ & $-0.85^{*}$ \\
\hline RTD & $-0.90^{*}$ & $0.83^{*}$ & $0.60^{*}$ & $-0.76^{*}$ \\
\hline RUE & $-0.89^{*}$ & $0.90^{*}$ & $0.74^{*}$ & $0.25^{*}$ \\
\hline HUE & -0.09 & $0.50^{*}$ & $0.46^{*}$ & 0.15 \\
\hline
\end{tabular}

*Significance at $\mathrm{P}=0.05$

Table.6 Correlation coefficient of agrometeorological indices with yield and yield attributes during vegetative and reproductive phase under different growing environments

\begin{tabular}{|c|c|c|c|c|c|c|c|c|c|}
\hline Particulars & $\begin{array}{c}\text { No. of } \\
\text { effective } \\
\text { tillers }\end{array}$ & $\begin{array}{c}\text { Spike } \\
\text { length } \\
(\mathrm{cm})\end{array}$ & $\begin{array}{c}\text { No. of } \\
\text { spikelets/ } \\
\text { spike }\end{array}$ & $\begin{array}{l}\text { No. of } \\
\text { grain/ } \\
\text { spike }\end{array}$ & $\begin{array}{c}\text { Test } \\
\text { weight } \\
\text { (g) }\end{array}$ & $\begin{array}{c}\text { Grain } \\
\text { yield } \\
\text { (q/ha) }\end{array}$ & $\begin{array}{c}\text { Biological } \\
\text { yield } \\
\text { (q/ha) }\end{array}$ & $\begin{array}{c}\text { Straw } \\
\text { yield } \\
(\mathbf{q} / \mathbf{h a})\end{array}$ & HI \% \\
\hline \multicolumn{10}{|c|}{ Vegetative phase } \\
\hline GDD & $0.22 *$ & 0.13 & 0.09 & 0.15 & 0.12 & $0.36^{*}$ & $0.30 *$ & 0.01 & $0.31^{*}$ \\
\hline HTU & $0.18 *$ & 0.11 & 0.07 & 0.13 & 0.07 & $0.31 *$ & $0.24 *$ & -0.02 & $0.27 *$ \\
\hline PTU & $0.21 *$ & 0.13 & 0.09 & 0.15 & 0.12 & $0.35 *$ & $0.30 *$ & 0.02 & $0.29 *$ \\
\hline RTD & -0.04 & -0.03 & -0.02 & -0.03 & -0.03 & -0.08 & -0.06 & 0.01 & -0.06 \\
\hline RUE & $0.36^{*}$ & -0.05 & -0.03 & 0.02 & $0.27 *$ & $0.28^{*}$ & $0.32 *$ & 0.15 & $0.18^{*}$ \\
\hline HUE & $0.25 *$ & -0.09 & -0.06 & -0.02 & $0.18^{*}$ & 0.14 & $0.20 *$ & 0.14 & 0.05 \\
\hline \multicolumn{10}{|c|}{ Reproductive phase } \\
\hline GDD & -0.06 & -0.02 & -0.02 & -0.03 & -0.03 & -0.09 & -0.04 & 0.06 & -0.11 \\
\hline HTU & -0.15 & -0.07 & -0.04 & -0.07 & -0.09 & $-0.22 *$ & -0.15 & 0.05 & $-0.23^{*}$ \\
\hline PTU & -0.11 & -0.05 & -0.04 & -0.06 & -0.05 & $-0.17 *$ & -0.10 & 0.07 & $-0.19 *$ \\
\hline RTD & 0.04 & 0.01 & 0.00 & 0.01 & 0.02 & 0.04 & 0.01 & -0.04 & 0.06 \\
\hline RUE & $0.74 *$ & 0.13 & -0.11 & 0.03 & $0.50 *$ & $0.72 *$ & $0.73^{*}$ & $0.26^{*}$ & $0.50 *$ \\
\hline HUE & $0.61 *$ & 0.01 & $-0.19^{*}$ & -0.11 & $0.40 *$ & $0.46^{*}$ & $0.50 *$ & $0.22 *$ & $0.31 *$ \\
\hline
\end{tabular}

*Significance at $\mathrm{P}=0.05$ 
Table.7 Correlation coefficient of soil moisture with canopy temperature $\left({ }^{\circ} \mathrm{C}\right)$ during vegetative and reproductive phase under different growing environments

\begin{tabular}{|c|c|c|c|c|}
\hline Particulars & \multicolumn{3}{|c|}{ Canopy temperature $\left({ }^{\circ} \mathbf{C}\right)$} & \multirow[b]{2}{*}{$D_{4^{-}} 19^{\text {th }}$ Dec. } \\
\hline $\begin{array}{c}\text { Soil Moisture } \\
(\%)\end{array}$ & $D_{1}-3^{\text {rd }}$ Nov. & $D_{2}-18^{\text {th }}$ Nov. & $D_{3^{-}} 3^{\text {rd }}$ Dec. & \\
\hline \multicolumn{5}{|c|}{ Vegetative phase } \\
\hline $0-15 \mathrm{~cm}$ & $-0.48 *$ & $-0.59 *$ & $-0.60 *$ & $-0.55^{*}$ \\
\hline $15-30 \mathrm{~cm}$ & $-0.25 *$ & -0.13 & -0.02 & -0.01 \\
\hline $30-60 \mathrm{~cm}$ & $-0.60 *$ & $-0.72 *$ & $-0.67 *$ & $-0.65^{*}$ \\
\hline $60-90 \mathrm{~cm}$ & $-0.47 *$ & $-0.51^{*}$ & $-0.52 *$ & $-0.50 *$ \\
\hline \multicolumn{5}{|c|}{ Reproductive phase } \\
\hline $0-15 \mathrm{~cm}$ & $0.55^{*}$ & $0.38 *$ & $0.49 *$ & $0.36 *$ \\
\hline $15-30 \mathrm{~cm}$ & -0.01 & 0.05 & -0.12 & $0.28 *$ \\
\hline $30-60 \mathrm{~cm}$ & 0.02 & 0.13 & -0.11 & $0.32 *$ \\
\hline $60-90 \mathrm{~cm}$ & 0.10 & $0.32 *$ & $0.19 *$ & $0.18 *$ \\
\hline
\end{tabular}

*Significance at $\mathrm{P}=0.05$

\section{Correlation studies between agrometeorological indices with yield and yield attributes}

All the agrometeorological indices like GDD, HTU, PTU, RUE and HUE were positively associated with yield attributes during vegetative phase except HTU with straw yield, RUE with spike length, no. of spikelets per spike and HUE with spike length, no. of spikelets per spike and no. of grains per spike (Table 6).

The RTD was negatively associated with yield attributes during vegetative phase except with straw yield. Among agrometeorological indices GDD, HTU and PTU were negatively associated with yield attributes except with straw yield during reproductive phase. RTD, RUE and HUE were positively correlated with yield attributes except RTD with straw yield, RUE with no. of spikelets per spike, HUE with no. of spikelets per spike and no. of grains per spike during reproductive phase. The association of agrometeorological indices like GDD, HTU, PTU, RTD, RUE and HUE with yield attributes was higher during vegetative phase than reproductive phase except the association with straw yield among yield studies.

\section{Correlation studies between soil moisture and canopy temperature}

The association of soil moisture content at varying depths with canopy temperature under different growing environments is shown during vegetative and reproductive phase (Table 7). The association of soil moisture content at varying depths viz. 0-15, $15-30,30-60$ and $60-90 \mathrm{~cm}$ with canopy temperature under varying growing environments was shown negatively significant relationship during vegetative phase.

During reproductive phase, soil moisture in 015 DAS layer had shown significantly positive correlation with the entire growing environment. Soil moisture at 15-30 DAS had negative correlation with canopy temperature of $\mathrm{D}_{1}$ and $\mathrm{D}_{3}$ sown crop whereas, $\mathrm{D}_{2}$ and $\mathrm{D}_{4}$ sown crop shown positive relationship. Soil moisture in deep layers (30-60 and 60-90 cm) had positive association with barley canopy temperature except in layer $30-60 \mathrm{~cm}$ with $\mathrm{D}_{3}$ sown crop. The association of soil moisture content at varying depths with canopy temperature under different growing environments was higher during vegetative phase than reproductive phase. 
In conclusion, the data obtained from this study in semi-arid regions of Haryana could be useful for barley agro-meteorologists, agronomists, breeders and grain producers in order to increase grain yield. Correlation analysis mirrored the strength of the relationship among agronomic and weather parameters in barley cultivars. These findings raise the finger towards yield perspective that had positive association with growth parameters during vegetative and reproductive phase.All growth parameters were shown significant correlation during reproductive phase as compare to vegetative phase with weather parameters. The agrometeorological indices like GDD, HTU, PTU, RTD, and RUE were highly correlated during reproductive phase and HUE at vegetative phase with weather parameters. Maximum temperature, BSS and RH (M) had positive correlation while minimum temperature, RH (E), WS, PE and RF had a negative relationship with yield and its attributes except straw yield during reproductive phase. The association of growth parameters with agrometeorological indices and yield attributes with agrometeorological indices was higher at vegetative phase than reproductive phase. Canopy temperature was found significant relationship with soil moisture of depth 0-15 and 15-30 cm during both the barley crop seasons under different growing environments.

\section{References}

Anonymous,2017, Crop wise area, average yield and production of major crops in Haryana for the year 2013-14 to 2017-18 (Anticipated) and target of 2018-19, http://agriharyana.gov.in/assets/ images/whatsnew/Five_Year_AYP_Targe ted_2016-17_N_Ek_Patti.pdf

Campbell, W.F., Wagenet, R.J., Bamatraf, A.F. and Turner, D.L., 1980. Path Coefficient Analysis of Correlation Between Stress and Barley Yield Components 1. Agronomy Journal, 72(6): 1012-1016.

CARPICI, E.B. and Celik, N., 2012. Correlation and path coefficient analyses of grain yield and yield components in two-rowed of barley (Hordeum vulgare convar. distichon) varieties. Notulae Scientia Biologicae, 4(2): 128-131.

Ehdaie, B. and Waines, J.G. 1993. Variation in water-use efficiency and its components in wheat: I. Well-watered pot experiment. Crop Science, 33(2): 294-299.

Hadjichristodoulou, A. 1983. Dual purpose barley. MPKV; Maharastra.

Kirby, E.J.M., Appleyard, M. and Fellowes, G. 1985. Effect of sowing date and variety on main shoot leaf emergence and number of leaves of barley and wheat. Agronomie, 5(2): 117-126.

Panse, V. G. and P. V. Sukhatme. 1985. Statistical methods for agricultural research workers. IInd Edition, ICAR, New Delhi.

Shamim, M.O.H.A.M.M.A.D., Devendra, S., Gangwar, K.K. and Vipin, K., 2013.Agrometeorological indices in relation to phenology, biomass accumulation and yield of rice genotypes under Western Plain zone of Uttar Pradesh. J. Agrometeorol, 15(2): 50-57.

Shepherd, K.D., Cooper, P.J.M., Allan, A.Y., Drennan, D.S.H. and Keatinge, J.D.H. 1987. Growth, water use and yield of barley in Mediterranean-type environments. The Journal of Agricultural Science, 108(2): 365-378.

\section{How to cite this article:}

Karan Chhabra, Mahendra Bele, Raj Singh and Anil Kumar. 2019. Analysis and Correlation among Agronomic and Weather Parameters in Barley (Hordeum vulgare L.) Cultivars under Semi-Arid Regions of Haryana, India. Int.J.Curr.Microbiol.App.Sci. 8(05): 1497-1505. doi: https://doi.org/10.20546/ijcmas.2019.805.172 